\title{
Nota Farmacológica: Tiazolinedionas ("glitazonas") y riesgo cardiovascular
}

\author{
Tiazolinediones ("glytazones") and cardiovascular risk
}

Marcela Botargues* y Mariela Barani**

\begin{abstract}
Resumen
Las tiazolinedionas son una opción de tratamiento en los pacientes con diabetes tipo 2 cuando no se han alcanzado las metas con los tratamientos de primera línea (metformina y sulfonilureas). Este artículo describe y discute la evidencia sobre la eficacia y la seguridad de las tiazolinedionas (rosiglitazona y pioglitazona).

\section{Abstract}

Tiazolinediones are a treatment option to be added in patients with type 2 diabetes who have not get the goals when treated with first line treatments (metformin and sulfonylureas). This article describes and discuss the evidence about efficacy and safety of two tiazolinediones (rosiglitazone and pioglitazone).
\end{abstract}

Palabras clave: tiazolinedionas, rosiglitazona, pioglitazona, riesgo cardiovascular, diabetes tipo 2 . Key words: tiazolinediones, rosiglitazone, pioglitazone, cardiovascular risk, type 2 diabetes.

Botargues M, Barani M. Tiazolinedionas ("glitazonas") y riesgo cardiovascular. Evid. actual. práct. ambul; 10(3): 85-86, mayjun.2007.

\section{Introducción}

En la nota farmacológica publicada en el volumen 10 Nro 1 pág. 29-30 de EVIDENCIA, se comentó la actualización del algoritmo para el abordaje de los pacientes con Diabetes Mellitus tipo 2. El mismo propone que en pacientes que no respondan al tratamiento de primera línea (cambios de estilo de vida más metformina) se agregue al tratamiento una de las siguientes tres opciones farmacológicas: sulfonilureas (opción más económica) insulina (especialmente indicada en pacientes con hemoglobina glicosilada o $\mathrm{HbA} 1 \mathrm{c}$ mayor a $8,5 \mathrm{~g} / \mathrm{dL}$ ) o tiazolinedionas ("gliazonas").

Recientemente se han publicado los resultados de un metaanálisis que reportó un aumento de la incidencia de infarto agudo de miocardio (IAM) en pacientes que recibían rosiglitazona. El objetivo de la presente revisión es evaluar el estado de conocimiento sobre los efectos cardiovasculares a largo plazo de esta familia de drogas.

\section{Rosiglitazona}

En el metaanálisis mencionado anteriormente ${ }^{1}$ fueron agrupados 42 estudios (con un total de 27847 pacientes) con las siguientes características: habían comparado rosiglitazona contra placebo durante por lo menos 24 semanas y habían evaluado los efectos de las droga sobre la incidencia de infarto agudo de miocardio (IAM) y muerte de origen cardiovascular.

Se observó un aumento significativo del riesgo de IAM (OR: $1,43$; IC95\%; $1,03-1,98)$ y no significativo de la muerte de origen cardiovascular (OR:1,64; IC95\%: 0,98-2,74) en pacientes con una edad promedio de 56 años y con una $\mathrm{HbA1c}$ de $8,2 \mathrm{~g} / \mathrm{dL}$ que habían tomado rosiglitazona durante 24 semanas o más.

Algunas de las debilidades de este estudio estudio son la presencia de pocos eventos (158 IAM y 61 muertes cardiovasculares) y que el reporte de datos surge de los estudios publicados y no del seguimiento de los pacientes, con la consiguiente dificultad del análisis del tiempo al evento y de la evolución temporal de los riesgos. Dentro de las fortalezas se rescata el intento de incluir estudios no publicados, el esfuerzo de analizar puntos finales duros como IAM y muerte cardiovascular, y la inclusión de estudios que hubieran comparado la droga contra placebo².

\section{Pioglitazona}

El ProActive ${ }^{3}$ es un estudio prospectivo, doble ciego, controlado y aleatorizado, diseñado con el objetivo de evaluar el rol de la pioglitazona en la prevención secundaria de enfermedad macrovascular en pacientes diabéticos tipo 2. En el mismo participaron 5238 pacientes de 35 a 75 años. Los pacientes fueron asignados a pioglitazona ( 15 a $45 \mathrm{mg}$ según tolerancia) o placebo, conservando además la terapia hipoglucemiante que ya venían recibiendo (dieta sola más ejercicio, hipoglucemiantes orales y/o insulina) con el fin de lograr una $\mathrm{HbA} 1 \mathrm{c}$ menor a $6,5 \mathrm{~g} / \mathrm{dL}$ y optimizar el control de los lípidos y la tensión arterial. Los pacientes incluídos tenían una $\mathrm{HbA} 1 \mathrm{c}$ mayor a $6,5 \mathrm{~g} / \mathrm{dL}$ y el antecedente de enfermedad macrovascular (IAM o accidente cerebrovascular o ACV) en los seis meses previos a la aleatorización. Fueron excluidos los pacientes que recibían monoterapia con insulina, tenían antecedentes de gangrena, úlceras isquémicas o dolor de reposo, insuficiencia cardíaca clase II, III y IV según la clasificación de Nueva York*, recibían hemodiálisis, tenían insuficiencia hepática o indicación de cirugía de revascularización miocárdica o arterial periférica.

El resultado final primario fue una combinación de mortalidad por todas las causas, IAM no fatal, ACV, síndrome coronario agudo, intervención quirúrgica o endovascular de las arterias coronarias o de los miembros inferiores y amputación por debajo de la rodilla. El resultado final secundario fue una combinación de tiempo a la muerte por cualquier causa, o al IAM o al ACV (lo que ocurriera primero); muerte cardiovascular; y los componentes individuales del resultado final primario compuesto.

Luego de tres años de seguimiento se observó una disminución no significativa del resultado final primario (HR* 0,9 IC95\%: 0,8-1,2). En cambio, sí se objetivó una diferencia estadísticamente significativa al analizarse el resultado final secundario. El grupo tratado con pioglitazona mostró una reducción absoluta del mismo de $1,9 \%(11,6 \%$ vs. $13,5 \%$, $\mathrm{p}=0,27)$ y un numero de pacientes necesarios a tratar para evitar un evento (NNT*) de 53. La pioglitazona se asoció con un aumento absoluto del $3 \%$ de la incidencia de insuficiencia cardíaca $(11 \%$ vs. $8 \%, p<0,0001)$ y de un aumento del $2 \%$ de la incidencia de hospitalizaciones por insuficiencia cardíaca $(p=0,003)$. Además, la incidencia de edemas periféricos ( $\sin$ insuficiencia cardíaca) fue de $22 \%$ en el grupo tratamiento comparado con el $13 \%$ del grupo placebo.

\footnotetext{
* Servicio de Medicina Familiar y Comunitaria del Hospital Italiano de Buenos Aires. maria.botargues@ hospitalitaliano.org.ar

** Servicio de Medicina Familiar y Comunitaria del Hospital Italiano de Buenos Aires. mariela.barani@hospitalitaliano.org.ar
} 
Este estudio es metodológicamente adecuado y fue diseñado con la idea de demostrar los beneficios de la pioglitazona en la prevención secundaria de la enfermedad macrovascular (cardíaca y periférica) en pacientes diabéticos. Sin embargo, en el resultado final primario no se observaron diferencias significativas entre los que recibieron pioglitazona y los que no. Por lo tanto, todavía no hay evidencia suficiente que avale el uso de la pioglitazona para prevenir enfermedad macrovascular. De todos modos, podría haber sido más adecuado evaluar los puntos finales vasculares periféricos como un resultado final secundario y no formando parte del resultado final primario compuesto (lo cual puede haber diluído los resultados a favor del tratamiento). El resultado final secundario sí mostró beneficios: una reducción del $2 \%$ de la incidencia de muerte, IAM o ACV asociada al tratamiento con pioglitazona. Sin embargo, contrasta con el incremento absoluto del $3 \%$ de la incidencia de insuficiencia cardíaca, que sorprendentemente no se vio reflejado en un aumento de la mortalidad.

\section{Conclusiones}

Teniendo en cuenta que las "glitazonas" están recomendadas como tratamiento de elección cuando no se logran las metas deseadas con las drogas del primera línea (metformina y sul- fonilureas) y conociendo que la enfermedad cardiovascular es la primera causa de muerte en diabéticos, se impone determinar la relación de las "glitazonas" con el riesgo cardiovascular. En el razonamiento médico de la práctica diaria es muy frecuente tomar decisiones en base a un criterio fisiológico puro basado en que si la glucemia y la hemoglobina glicosilada por encima de ciertas metas causan daño a largo plazo y estas drogas son capaces de provocar su reducción, ergo, se supone que su administración causará un efecto beneficioso para la salud. Sin embargo, poco conocemos acerca de cómo influyen sobre los eventos en el largo plazo los edemas, la anemia, el aumento de peso y los cambios lipídicos; y en definitiva, si el balance final será beneficioso.

Una reflexión aparte merecen los requisitos para aprobar el uso de una droga nueva y cuál es el tiempo suficiente de seguimiento de la misma para conocer su seguridad.

Hoy no sabemos si la rosiglitazona es capaz de aumentar o disminuir el riesgo cardiovascular y debemos esperar el resultado de nuevos estudios para determinarlo. Respecto de la pioglitazona, la evidencia tampoco es concluyente ya que si bien demostró disminuir la incidencia de muerte, IAM o ACV en un $2 \%$ en diabéticos que ya habían tenido un evento cardiovascular, también aumentó un 3\% la incidencia de insuficiencia cardíaca.

\section{Referencias}

1. Effect of Rosiglitazone on the Risk of Myocardial Infarction and Death of Cardiovascular Causes. Nissen and Wolski. NEJM 2007; $356: 2457-71$.

2. Rosiglitazone and Cardiovascular Risk. Bruce M. Psaty, M.D., Ph.D., and Curt D. Furberg, M.D., Ph.D. NEJM 2007, Volume 356:2522-2524.

3. Secondary prevention of macrovascular events in patients with type 2 diabetes in the PROactive Study (PROspective pioglitAzone Clinical Trial In macroVascular Events): a randomised controlled trial. The Lancet, Volume 366, Issue 9493, 8 October 2005-14 October 2005, Pages 1279-1289.

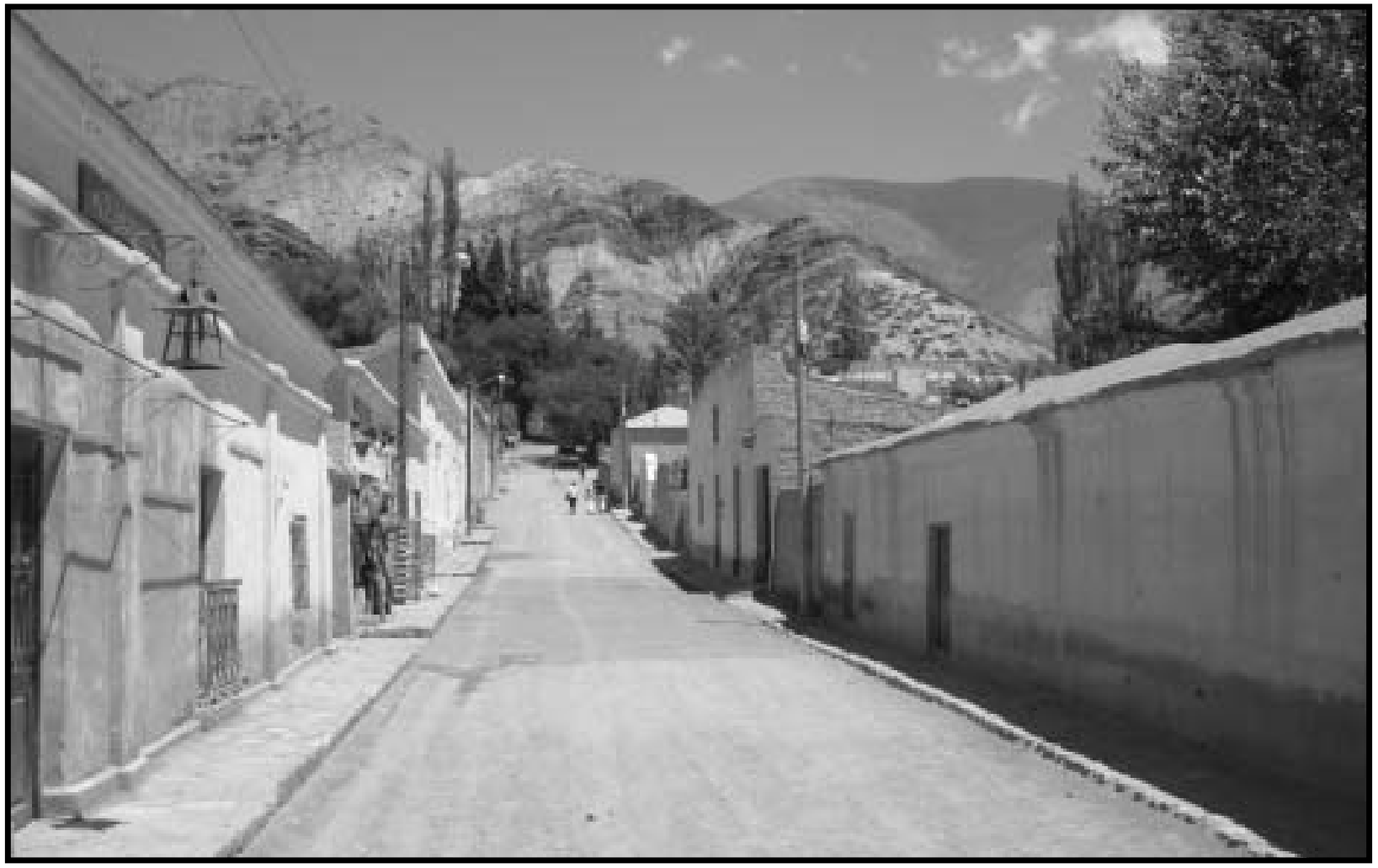

Purmamarca, Jujuy, Argentina. Cortesía: Fotos: Guadalupe Alessia. 